\title{
Cardiovascular clinical applications of PET/MRI
}

\author{
Osman Ratib - René Nkoulou $\cdot$ Markus Schwaiger
}

Received: 10 December 2012/ Accepted: 24 January 2013/Published online: 16 February 2013

(C) Italian Association of Nuclear Medicine and Molecular Imaging 2013

\begin{abstract}
Cardiac PET imaging is well established as the reference modality for quantitative evaluation of myocardial perfusion and for the detection of ischemic alterations in coronary blood flow using radiolabeled PET tracers such as ${ }^{13} \mathrm{~N}$-ammonia, ${ }^{82} \mathrm{Rb}$ and ${ }^{15} \mathrm{O}$-labeled water. While these techniques have served as the gold standard for clinical research and advanced evaluation of ischemic disease, the emergence of ${ }^{18} \mathrm{~F}$-labeled perfusion tracers may, in the future, bring cardiac PET imaging into the mainstream of clinical imaging for assessment of coronary artery disease (CAD). FDG PET imaging has also served as a standard for objective assessment of the extent of myocardial viability after myocardial infarction and remains the most accurate technique for predicting recovery of myocardial function after reperfusion and for selecting patients who may benefit from revascularization procedures. Cardiac MRI has concurrently evolved as a technique that provides high-definition dynamic images of the heart and great vessels, surpassing echocardiography for accurate evaluation of cardiac function. Recent developments have also shown its ability to identify areas of reduced myocardial perfusion after gadolinium injection at rest and during pharmacological stress, thus promoting stress cardiac MRI as an alternative modality for detection of CAD. Furthermore, delayed accumulation of contrast media in scar and fibrotic
\end{abstract}

\section{O. Ratib $(\varangle) \cdot$ R. Nkoulou}

Division of Nuclear Medicine and Molecular Imaging, University Hospital of Geneva, 24, rue Micheli-du-Crest, 1205 Geneva, Switzerland

e-mail: osman.ratib@hcuge.ch

M. Schwaiger

Nuklearmedizinische Klinik und Poliklinik Klinikum rechts der Isar der Technischen Universität München,

Munich, Germany tissue allows for differentiation of viable and non-viable tissue after myocardial infarction. PET and MRI, relying on different biological and physiological mechanisms, are truly complementary in their ability to detect stressinduced myocardial ischemia and tissue viability. This complementary ability of the two modalities opens up new perspectives in the assessment of cardiovascular disease. It is therefore foreseeable that the emergence of hybrid devices combining PET and MR modalities will lead to the development of new diagnostic protocols offering better diagnostic accuracy and allowing more objective assessment of cardiovascular disease. These applications may well extend beyond the scope of CAD and allow the technique to enter other areas: the evaluation of vascular plaques, neurotransmitter alterations and mechanisms of angiogenesis, the differentiation of different gene expression phenotypes, and the follow-up of stem cell therapy.

Keywords PET/MRI · Hybrid imaging · Nuclear medicine $\cdot$ Molecular imaging

\section{Introduction}

Over the past decade the evolution of PET/CT has gained a certain momentum in clinical applications, oncology in particular. As an immediate consequence of this growth a new generation of PET/CT and now hybrid PET/MR scanners has emerged and been adopted by the medical community at large, allowing the use of this technology to extend well beyond the sphere of the early adopters in academic institutions. The availability of hybrid PET and multi-detector CT scanners that allow high-definition dynamic imaging of the heart and coronary vessels has opened up a new perspective on cardiac imaging, allowing 
combined anatomical and functional evaluation of coronary disease and cardiac function [1] (Fig. 1). Concurrently, MRI technology has evolved rapidly, leading to new imaging protocols that allow dynamic acquisition of cardiac images for functional analysis of regional myocardial wall motion and ventricular function. First-pass imaging of gadolinium contrast media through the myocardium was shown to allow differentiation between normal and hypoperfused myocardium [2]. Changes in perfusion pattern between rest and pharmacological stress allow the depiction of areas with stress-induced ischemia caused by underlying hemodynamically significant coronary artery disease (CAD). While stress cardiac MRI has gained wider acceptance in clinical routine, its use for the detection of mild to moderate ischemic alterations may still need to be fully evaluated, in comparison with PET perfusion scans.

Combining PET and MRI can, therefore, probably be expected to offer the added value of the best diagnostic performance of the two modalities used in conjunction. The high temporal resolution of MRI images, allowing superior soft tissue differentiation, exceeds the possibilities offered by multidetector $\mathrm{CT}$. However, in terms of coronary artery visualization and the identification of segmental stenosis and vascular plaques, MRI images remain limited.

On another hand, FDG PET remains the reference standard for the assessment of myocardial viability after myocardial infarction. Delayed retention of gadolinium on MRI images has been shown to allow localization of scar and fibrotic tissue and therefore the identification of nonviable tissue [3]. While it provides accurate morphological identification of scar tissue it lacks the ability to accurately measure and quantify the amount of metabolic activity of residual viable myocardium. Upregulation of ${ }^{18}$ F-FDG uptake in ischemically compromised myocardium has been shown to provide prognostic information independently of infarct size. Therefore, it is also anticipated that the combination of PET and MRI for assessment of myocardial viability will provide better diagnostic accuracy and a more quantitative evaluation of regional myocardial viability, offering a better prediction of potential improvement of ventricular function following revascularization procedures (Fig. 2).

The aim of this paper is to review the current state of the art in cardiac PET and cardiac MRI and to report early observations and clinical applications of new hybrid scanners combining these two complementary modalities. It also highlights other potential applications of hybrid PET/MR, beyond the scope of CAD, that may see the technique entering other areas: the evaluation of vascular plaques and, through the development of new radiotracers allowing the assessment of alterations in neurotransmitters, the evaluation of mechanisms of angiogenesis, and even

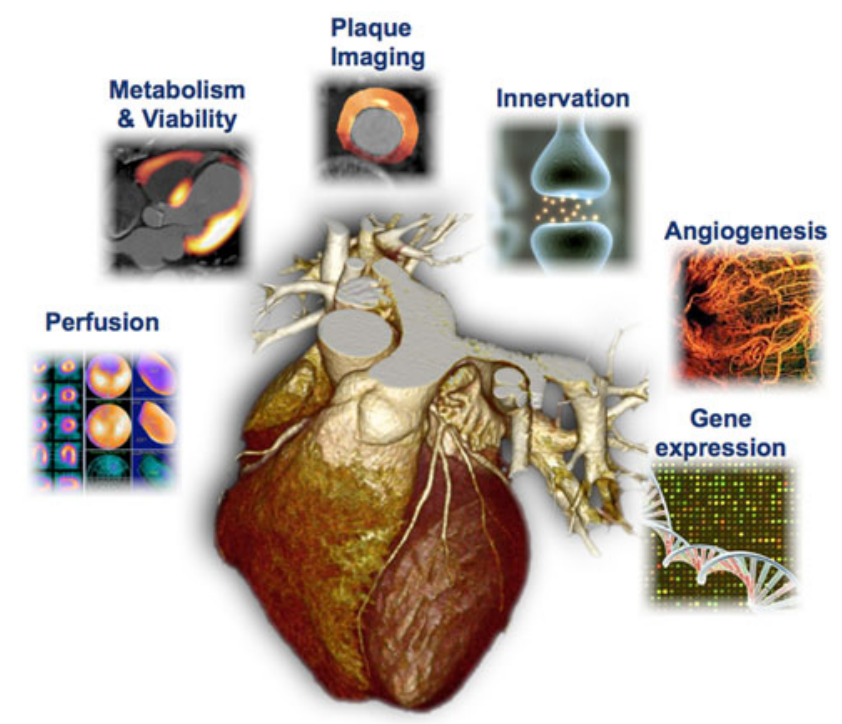

Fig. 1 Potential applications of PET molecular imaging capabilities open up new perspectives in the assessment of many different biological and functional processes, and thus the prospect of new tools for the detection and diagnosis of different diseases

the evaluation of different gene expressions; it could also allow the follow-up of stem cell therapy.

\section{Technical advances in hybrid PET/MR}

Different designs combining PET and MR devices are being developed by different vendors. These solutions include systems able to co-register studies obtained from two different devices (where the patient is moved on a shuttle-bed between two separate examination rooms), co-planar devices that combine PET and MR scanners located around the same bed in a single room, and, most recently, the integration of PET and MR devices into a single scanner [4]. Separate co-planar devices require sequential acquisition of images from each modality, while integrated systems have the potential to acquire images from both modalities simultaneously [5, 6]. While simultaneous acquisition has limited advantages for oncology investigations, it may be important in functional imaging applications, for example in pharmacological stress and neurology [7].

The first generation of integrated PET/MR scanners was designed with avalanche photodiode (APD) technology and, in performance terms, these scanners are comparable to state-of-the-art PET/CT scanners. APDs can be replaced by higher performance solid-state digital detectors providing higher sensitivity and temporal resolution allowing time-of-flight imaging. 

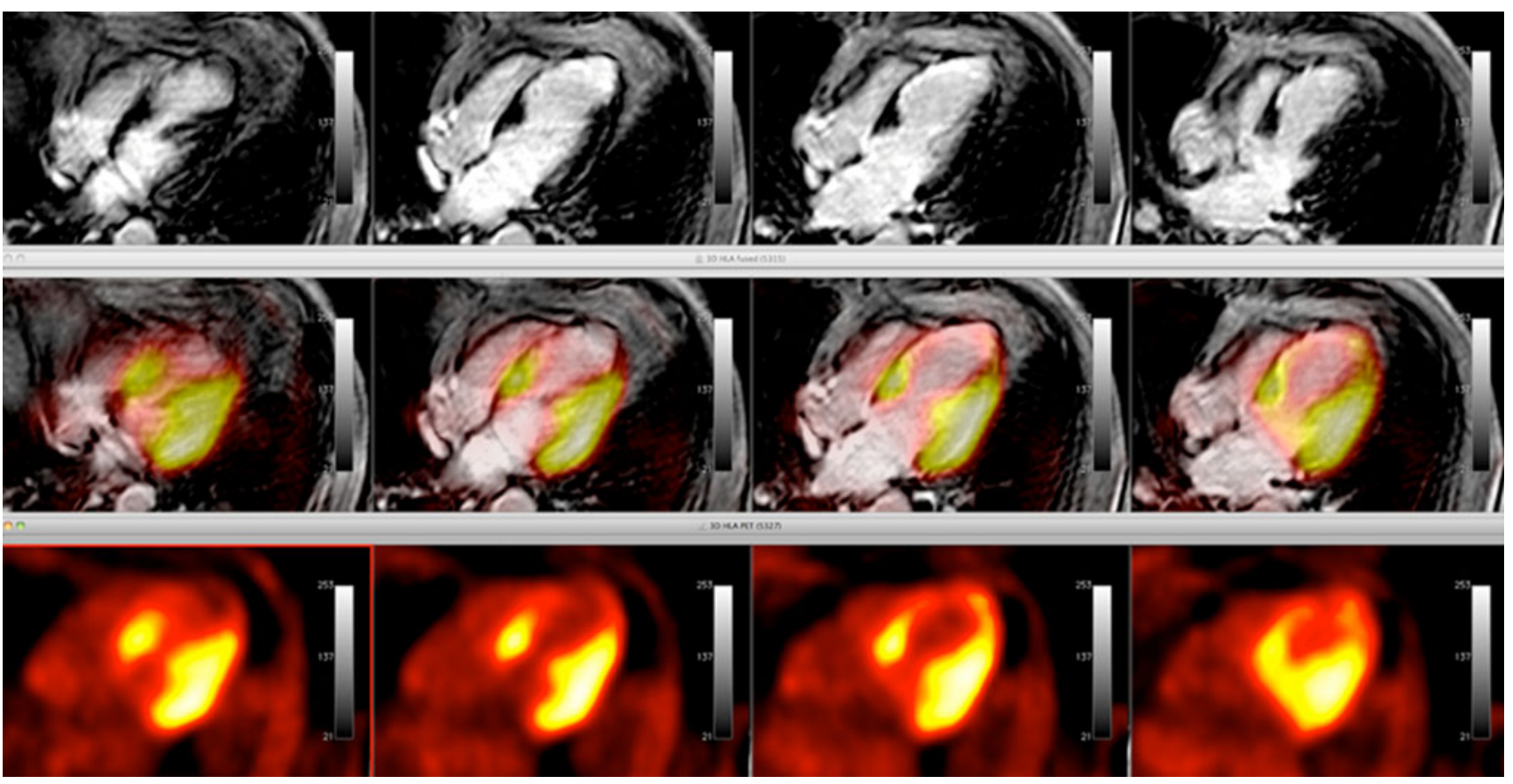

Fig. 2 Example of horizontal long axis plane views of MR (top), PET (bottom) and fused (middle) images obtained from a patient with an apico-septal myocardial infarct. The patterns of wall thinning and

\section{PET and MRI in coronary artery disease}

The paradigm of CAD detection has changed over the years, with anatomical characterization of stenotic plaques by invasive coronary angiogram making way for interest in the additional evidence provided by stress-induced coronary flow limitation and, more recently, interest in plaque morphology $[8,9]$.

Modern PET/CT systems used for CAD detection most commonly involve high-end multi-detector CT providing low-dose assessment of the coronary anatomy with excellent negative predictive values as compared to invasive coronary angiograms $[10,11]$. Hybrid cardiac imaging with radionuclide perfusion assessment complemented by coronary CT angiography is now clinically adopted for guiding vessel revascularization in cases of ischemia and for differentiating epicardial coronary stenosis from sub-epicardial disease when low hyperemic flow response suggests diffuse CAD [1]. However, the CT technique presents several limitations including the use of iodinated contrast media and ionizing radiation, as well as inconclusive assessment of heavily calcified vessels and irregular heart rate. These limitations prompted efforts to improve MR technology in order to provide a non-invasive alternative for assessment of the coronary anatomy. The diagnostic accuracy of non-contrast coronary MR angiography (CMRA) has increased steadily thanks to the use of navigator-guided whole-heart 3D acquisition pulse sequences tissue scarring seen on the MR images coincide with the areas of decreased glucose metabolism on the FDG PET images

with reported sensitivity and specificity of $78-96 \%$ and $68-96 \%$, respectively, in the detection of ICA luminal narrowing of at least $50 \%$ using $1.5 \mathrm{~T}$ systems; $3 \mathrm{~T}$ systems are expected to provide further improvements due to increased spatial resolution $[12,13]$. Despite some caveats including incomplete assessment of secondary coronary branches and time-consuming acquisitions, the high reliability of CMRA for ruling out left main or thee-vessel disease could undoubtedly benefit future hybrid PET/MR applications, especially bearing in mind the reduced sensitivity of relative perfusion evaluation in potential balanced ischemia and the difficult interpretation of reduced absolute flow (epicardial vs subepicardial disease) in patients with multi-vessel CAD [14].

There is now convincing evidence that CMRA is reliable in the assessment of hemodynamic consequences of moderate coronary stenosis. While allowing the visualization of late-stage response to ischemia as reversible wall motion abnormalities, first-pass imaging is also proving comparable to SPECT-based evaluation of stressinduced perfusion defects [2]. Although PET has consistently been considered superior to SPECT in this regard and is now widely recognized as the gold standard for perfusion evaluation, only a limited number of correlative studies comparing PET and MRI perfusion have been published $[15,16]$. Technical advantages of PET perfusion over CMRA include exquisite signal discrimination and low dependence on plane selection owing to 
inherently whole-heart 3D acquisition. More importantly, robust absolute flow quantification in $\mathrm{ml} / \mathrm{g} / \mathrm{min}$ is possible owing to near-linear extraction and predictable compartment modeling of perfusion tracers [17]. The possibility of evaluating perfusion defects in a sizable way by PET has been shown to have prognostic value, as it allows estimation of ischemic burden before revascularization and consideration of the implications of reduced coronary flow reserve [18].

In a comparative study, MRI showed insensitive depiction of coronary flow increase: in the range of 1.3, compared to 2.5 using PET [16]. PET myocardial perfusion faces several challenges including the need for a more optimal perfusion tracer displaying a high flow-independent extraction rate, a suitable half-life, and a low radiation burden. With ${ }^{1513} \mathrm{O}$-labeled water and $\mathrm{NH}_{3}$ needing an on-site cyclotron and ${ }^{82} \mathrm{Rb}$ generator, and thus having high operational costs, the newly proposed ${ }^{18} \mathrm{~F}$-labeled flurpiridaz perfusion tracer with 110 min half-life and myocardial trapping would allow widespread use of PET perfusion scans in clinical practice [19]. However, prolonged retention time in the myocardium necessitates higher radiotracer doses for repeated injections, as in stress/rest protocols. This requires proper adjustment of injected doses that can be significantly reduced with the newer generation of PET scanners leading to lower exposure dose comparable to myocardial perfusion SPECT studies. Combined PET/MR offers a series of potential advantages of in this setting:

- Complementary assessment of wall motion abnormalities and identification of regional and segmental hypokinesis, as well as wall thinning and scar tissue;

- Design of stress-only PET perfusion protocol, with rest perfusion evaluated by CMRA:

- Correlation of microcirculation with delayed contrastenhanced MR and tissue characterization (fibrosis) (Fig. 3).

\section{Assessment of myocardial viability}

Since the early evidence, in the 1970s, of potential functional recovery of akinetic myocardium-studies showed the effect of revascularization on left ventricular performance, augmentation of contraction in CAD by catecholamine stimulation, and post extra-systolic potentiation of contractility by atrial stimulation [20-22] - the role of preoperative imaging in predicting functional recovery has been emphasized in numerous meta-analyses. As a result, the ESC and AHA/ACC recommended pre-operative imaging, before revascularization, in heart failure and stable CAD patients (class IIA recommendation) [23]. Although SPECT and dobutamine stress echocardiography have both been traditionally used, PET/CT using ${ }^{18}$ F-FDG after metabolic homogenization and CMRA using contractile recruitment after low-dose dobutamine or discrimination of the extent of transmural late gadolinium enhancement are now the most established techniques. PET and CMRA provide complementary data with similar diagnostic performance [24, 25]. In the PARR-2 trial, randomizing patients to a clinical follow-up using ${ }^{18} \mathrm{~F}$-FDG PET for the assessment of viability did not show a significant overall benefit of including PET in patient management. However, this result has to be considered tempered by the high non-adherence to the PET recommendation, $25 \%$ in this study, and by the high percentage of cross-over with significant underpowering of the study, which highlights the difficulty in designing imaging clinical trials in such complex management plans [26]. The validity of viability assessment has also been stressed further by the more recent STICH sub-study trial that used less optimal SPECT and DSE tests as discriminators of viability [27]. When considering only PET and CMRA studies, a significant concern is the low specificity of both techniques in predicting contractile recovery after

Hybrid MPI Protocol

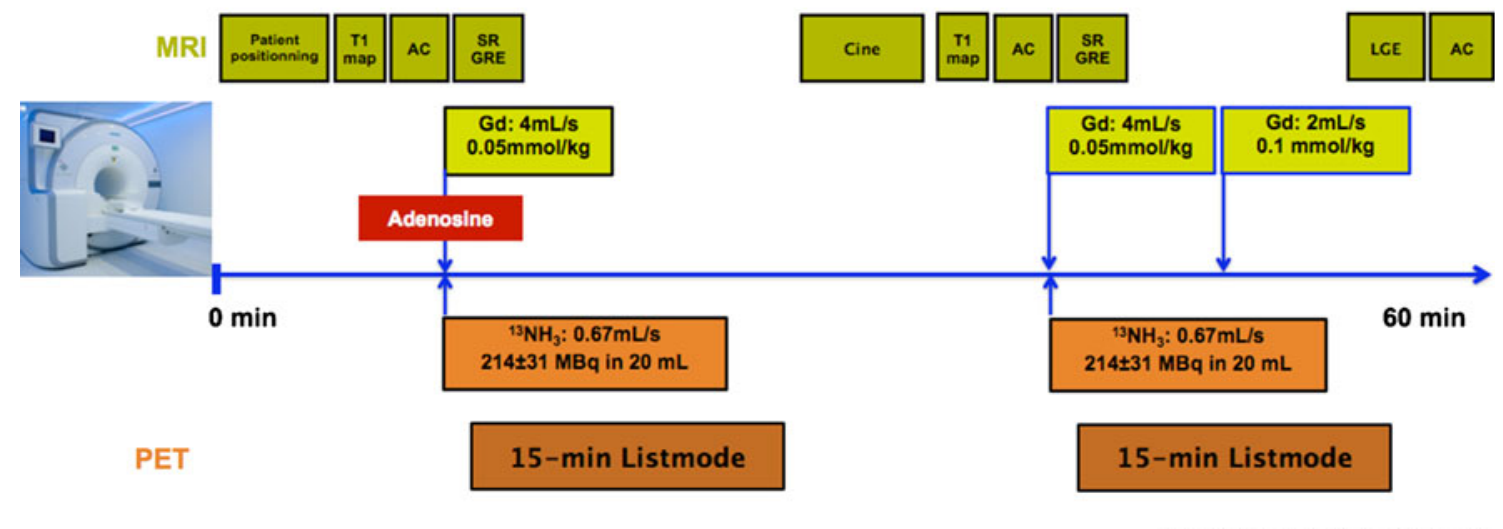

odoptod from Zhang. Nekollo. Schwaigor, TUM

Fig. 3 Schematic diagram of hybrid PET/MR myocardial perfusion imaging (MPI) during pharmacological stress and at rest 
Fig. 4 Viability study in a patient with large antero-apical myocardial infarct. a Delayed MR image showing late enhancement post-contrast injection of the antero-apical wall, b superimposed PET images showing increased ${ }^{18}$ F-FDG uptake in the apical area consistent with viable myocardium in hypermetabolic anaerobic state. Lower images show two levels across the long axis of the left ventricle: $\mathbf{c}$ a higher level showing viable myocardium, and $\mathbf{d}$ a lower level showing scar tissue with almost no metabolically viable myocardium
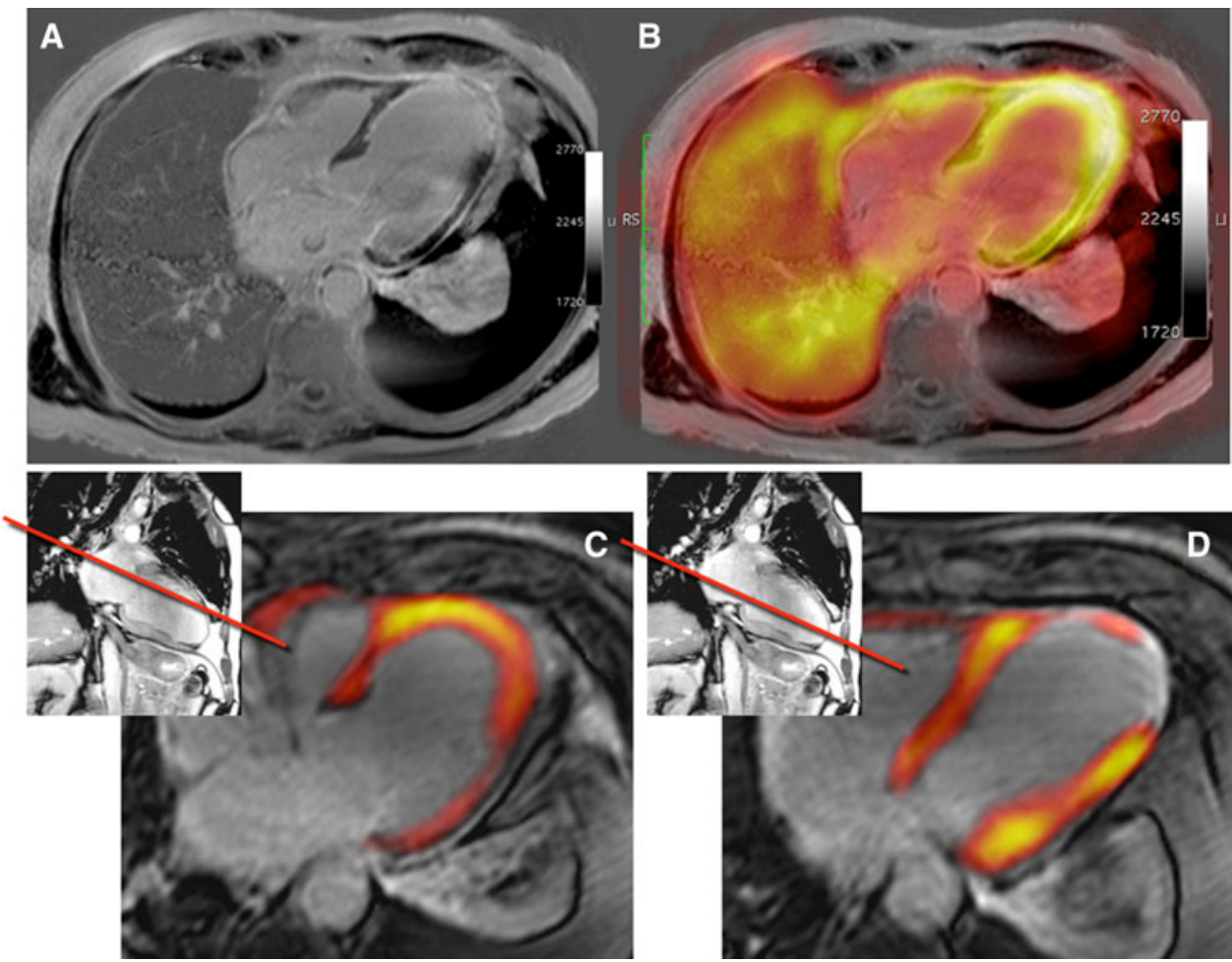

revascularization (one of three myocardial segments identified as viable failing to show further improvement upon revascularization). This highlights the fact that after a sustained ischemic condition, cardiomyocytes exhibit inhomogeneous adaptation/conditioning transformations including a shift in substrate utilization, modification of contractility, and contractile disorganization, with glucose metabolism probably being one of the last phases before cell disruption [28]. Focusing only on PET assessment of preserved glucose utilization or on CMRA to evaluate contractile reserve or cell integrity inherently carries limitations and acknowledgment of this continuum in cell survival states begs for more comprehensive protocols that include both techniques. Consensual diagnosis of transmural scar is often noted when PET and CMRA criteria are combined. However, whereas the poor prognostic value of CMRA in segments with late gadolinium enhancement (LGE) between 30 and $70 \%$ could be enhanced by associated PET assessment [29], PET proved relatively insensitive in detecting sub-endocardial scar $<25 \%$ of LGE. Integrating both approaches is also expected to provide better insight concerning the difficult issue of mild perfusion and metabolic match when nontransmural scar without residual myocardium at risk and ischemia are present. These combined PET and CMRA evaluations could of course be acquired sequentially, but the advantages of using new hybrid PET/MR systems include perfect co-registration and improved patient workflow (Fig. 4).

\section{Imaging vascular plaques}

Most acute cardiovascular events are consequences of rupture of atherosclerotic plaques that often do not initially result in significant luminal obstruction $[30,31]$. The causes of plaque instability and high rupture risk are believed to be related to inflammatory processes and the accumulation of macrophages in the lipid core of vessel plaques [32]. Most recent studies showed that intraplaque angiogenesis by proliferation of medial vasa vasorum has also been implicated in rapid plaque growth and plaque rupture. Therefore, non-invasive imaging of inflammation and angiogenesis within atherosclerotic lesions may be useful to predict future risk of plaque rupture and allow monitoring of antiatherosclerotic therapies [33]. While recent studies showed that PET imaging with ${ }^{18} \mathrm{~F}$-FDG could provide a non-invasive means of detecting inflammation in atherosclerotic lesions, its use in coronary arteries is limited due to the high glucose uptake of adjacent myocardium. Alternative tracers of plaque inflammation and vulnerability are being explored; they include ${ }^{18}$ F-galacto-RGD which binds with high specificity to $\propto v \beta 3$ integrin, a cell surface glycoprotein receptor that is highly expressed during angiogenesis [34]. While these early studies are promising, further studies are needed to characterize its uptake in plaques with high degrees of inflammation and neovascularization.

An alternative tracer recently evaluated for assessment and characterization of atherosclerotic plaques is 
${ }^{18} \mathrm{~F}$-labeled sodium fluoride $\left({ }^{18} \mathrm{~F}-\mathrm{NaF}\right)$. Preliminary studies suggest that ${ }^{18} \mathrm{~F}-\mathrm{NaF}$ uptake provides different information, relating to metabolically active calcific plaques and developing micro-calcification [35]. Prospective studies to determine the relationship between ${ }^{18} \mathrm{~F}-\mathrm{NaF}$ uptake, morphological plaque characteristics, and future cardiovascular events are still required in subjects with stable and unstable coronary disease.

While these new tracers still have to make their way into clinical use, several studies have reported the use of FDG PET imaging as a means of characterizing inflammatory activity within atherosclerotic plaques, and thus as a surrogate biomarker for detecting vulnerable plaques [36].

\section{Future perspectives of cardiac PET/MR imaging}

The upcoming generations of integrated hybrid PET/MR scanners offer an ideal combination of the imaging capabilities of MRI and PET for cardiac imaging. While cardiac MRI will continue to expand in cardiology, becoming increasingly widely adopted in clinical routine, the additional metabolic and functional information provided by PET tracers will provide additional value for specific subsets of patients who will benefit from the added diagnostic accuracy that PET can provide. The promising results of new tracers for better identification of ischemic myocardial alterations and viability as well as better assessment of high-risk atherosclerotic plaques will open up new perspectives and diagnostic strategies for cardiovascular diseases.

Furthermore, PET/MR is set to become the imaging modality of choice for the evaluation and follow-up of new therapeutic techniques aimed at tissue regeneration and myocardial repair. Several groups have already reported early results of PET imaging techniques for assessment of the kinetics of stem cell therapy in myocardial infarction. Cell labeling may offer new tools for monitoring the delivery, survival and migration of cells following dual labeling with iron particles, and reporter gene technologies may address cell localization and viability following cell transplantation. However, the specificity of MR and PET signals needs to be validated, in order to provide guidance in cell therapy. PET/MR is expected to play a major role in the validation process allowing longitudinal studies providing comprehensive structural and biological information.

\section{Conclusion}

The rapid adoption of hybrid PET/MR in clinical practice is mostly driven by oncology applications, although cardiac imaging applications will benefit greatly from the broader availability of these new hybrid devices. The widespread adoption of cardiac MRI studies in clinical routine has also contributed to the development of specific imaging protocols for a variety of cardiovascular applications and will also facilitate the acceptance of hybrid devices in clinical practice. The added value of metabolic PET imaging will certainly grow further with the availability of new specific PET tracers.

Conflict of interest None.

\section{References}

1. Flotats A, Knuuti J, Gutberlet M et al (2011) Hybrid cardiac imaging: SPECT/CT and PET/CT. A joint position statement by the European Association of Nuclear Medicine (EANM), the European Society of Cardiac Radiology (ESCR) and the European Council of Nuclear Cardiology (ECNC). Eur J Nucl Med Mol Imaging 38(1):201-212

2. Schwitter J, Wacker CM, van Rossum AC et al (2008) MR-IMPACT: comparison of perfusion-cardiac magnetic resonance with single-photon emission computed tomography for the detection of coronary artery disease in a multicentre, multivendor, randomized trial. Eur Heart J 29(4):480-489

3. Kim RJ, Wu E, Rafael A et al (2000) The use of contrastenhanced magnetic resonance imaging to identify reversible myocardial dysfunction. N Engl J Med 343(20):1445-1453

4. Balyasnikova S, Lofgren J, de Nijs R, Zamogilnaya Y, Hojgaard L, Fischer BM (2012) PET/MR in oncology: an introduction with focus on MR and future perspectives for hybrid imaging. Am J Nucl Med Mol Imaging 2(4):458-474

5. Zaidi H, Ojha N, Morich M et al (2011) Design and performance evaluation of a whole-body Ingenuity TF PET-MRI system. Phys Med Biol 56(10):3091-3106

6. Delso G, Furst S, Jakoby B et al (2011) Performance measurements of the Siemens mMR integrated whole-body PET/MR scanner. J Nucl Med 52(12):1914-1922

7. Stegger L, Martirosian P, Schwenzer N et al (2012) Simultaneous PET/MR imaging of the brain: feasibility of cerebral blood flow measurements with FAIR-TrueFISP arterial spin labeling MRI. Acta Radiol 53(9):1066-1072

8. De Bruyne B, Pijls NH, Kalesan B et al (2012) Fractional flow reserve-guided PCI versus medical therapy in stable coronary disease. N Engl J Med 367(11):991-1001

9. Stone GW (2012) In search of vulnerable plaque. Circ Cardiovasc Imaging 5(4):428-430

10. van Werkhoven JM, Bax JJ, Nucifora G et al (2009) The value of multi-slice-computed tomography coronary angiography for risk stratification. J Nucl Cardiol 16(6):970-980

11. Budoff MJ, Dowe D, Jollis JG et al (2008) Diagnostic performance of 64-multidetector row coronary computed tomographic angiography for evaluation of coronary artery stenosis in individuals without known coronary artery disease: results from the prospective multicenter ACCURACY (Assessment by Coronary Computed Tomographic Angiography of Individuals Undergoing Invasive Coronary Angiography) trial. J Am Coll Cardiol 52(21):1724-1732

12. Jahnke C, Paetsch I, Nehrke K et al (2005) Rapid and complete coronary arterial tree visualization with magnetic resonance imaging: feasibility and diagnostic performance. Eur Heart J 26(21): 2313-2319 
13. Nagata M, Kato S, Kitagawa K et al (2011) Diagnostic accuracy of 1.5-T unenhanced whole-heart coronary MR angiography performed with 32-channel cardiac coils: initial single-center experience. Radiology 259(2):384-392

14. Di Carli MF, Murthy VL (2011) Cardiac PET/CT for the evaluation of known or suspected coronary artery disease. Radiographics 31(5):1239-1254

15. Schwitter J, DeMarco T, Kneifel S et al (2000) Magnetic resonance-based assessment of global coronary flow and flow reserve and its relation to left ventricular functional parameters: a comparison with positron emission tomography. Circulation 101(23):2696-2702

16. Ibrahim T, Nekolla SG, Schreiber K et al (2002) Assessment of coronary flow reserve: comparison between contrast-enhanced magnetic resonance imaging and positron emission tomography. J Am Coll Cardiol 39(5):864-870

17. Nitzsche EU, Choi Y, Czernin J, Hoh CK, Huang SC, Schelbert HR (1996) Noninvasive quantification of myocardial blood flow in humans. A direct comparison of the $\left[{ }^{13} \mathrm{~N}\right]$ ammonia and the $\left[{ }^{15} \mathrm{O}\right]$ water techniques. Circulation 93(11):2000-2006

18. Herzog BA, Husmann L, Valenta I et al (2009) Long-term prognostic value of ${ }^{13} \mathrm{~N}$-ammonia myocardial perfusion positron emission tomography added value of coronary flow reserve. J Am Coll Cardiol 54(2):150-156

19. Nekolla SG, Reder S, Saraste A et al (2009) Evaluation of the novel myocardial perfusion positron-emission tomography tracer ${ }^{18} \mathrm{~F}-\mathrm{BMS}-747158-02$ : comparison to ${ }^{13} \mathrm{~N}$-ammonia and validation with microspheres in a pig model. Circulation 119(17): 2333-2342

20. Rees G, Bristow JD, Kremkau EL et al (1971) Influence of aortocoronary bypass surgery on left ventricular performance. N Engl J Med 284(20):1116-1120

21. Horn HR, LE Teichholz, Cohn PF, Herman MV, Gorlin R (1974) Augmentation of left ventricular contraction pattern in coronary artery disease by an inotropic catecholamine. The epinephrine ventriculogram. Circulation 49(6):1063-1071

22. Diamond GA, Forrester JS, de Luz PL, Wyatt HL, Swan HJ (1978) Post-extrasystolic potentiation of ischemic myocardium by atrial stimulation. Am Heart J 95(2):204-209

23. Wijns W, Kolh P, Danchin N et al (2010) Guidelines on myocardial revascularization. Eur Heart J 31(20):2501-2555

24. Allman KC, Shaw LJ, Hachamovitch R, Udelson JE (2002) Myocardial viability testing and impact of revascularization on prognosis in patients with coronary artery disease and left ventricular dysfunction: a meta-analysis. J Am Coll Cardiol 39(7): $1151-1158$
25. Kaandorp TA, Lamb HJ, van der Wall EE, de Roos A, Bax JJ (2005) Cardiovascular MR to access myocardial viability in chronic ischaemic LV dysfunction. Heart 91(10):1359-1365

26. Beanlands RS, Nichol G, Huszti E et al (2007) F-18-fluorodeoxyglucose positron emission tomography imaging-assisted management of patients with severe left ventricular dysfunction and suspected coronary disease: a randomized, controlled trial (PARR-2). J Am Coll Cardiol 50(20):2002-2012

27. Bonow RO, Maurer G, Lee KL et al (2011) Myocardial viability and survival in ischemic left ventricular dysfunction. N Engl J Med 364(17):1617-1625

28. Depre C, Vanoverschelde JL, Gerber B, Borgers M, Melin JA, Dion R (1997) Correlation of functional recovery with myocardial blood flow, glucose uptake, and morphologic features in patients with chronic left ventricular ischemic dysfunction undergoing coronary artery bypass grafting. J Thorac Cardiovasc Surg 113(2):371-378

29. Underwood SR, Bax JJ, vom Dahl J et al (2004) Imaging techniques for the assessment of myocardial hibernation. Report of a Study Group of the European Society of Cardiology. Eur Heart J 25(10):815-836

30. Ambrose JA, Tannenbaum MA, Alexopoulos D et al (1988) Angiographic progression of coronary artery disease and the development of myocardial infarction. J Am Coll Cardiol 12(1):56-62

31. Stone GW, Maehara A, Lansky AJ et al (2011) A prospective natural-history study of coronary atherosclerosis. N Engl J Med 364(3):226-235

32. Virmani R, Burke AP, Farb A, Kolodgie FD (2006) Pathology of the vulnerable plaque. J Am Coll Cardiol 47(8 Suppl):C13-C18

33. Laitinen I, Saraste A, Weidl E et al (2009) Evaluation of alphavbeta3 integrin-targeted positron emission tomography tracer ${ }^{18} \mathrm{~F}$-galacto-RGD for imaging of vascular inflammation in atherosclerotic mice. Circ Cardiovasc Imaging 2(4):331-338

34. Gaertner FC, Kessler H, Wester HJ, Schwaiger M, Beer AJ (2012) Radiolabelled RGD peptides for imaging and therapy. Eur J Nucl Med Mol Imaging 39(Suppl 1):S126-S138

35. Dweck MR, Chow MW, Joshi NV et al (2012) Coronary arterial ${ }^{18} \mathrm{~F}$-sodium fluoride uptake: a novel marker of plaque biology. J Am Coll Cardiol 59(17):1539-1548

36. Cocker MS, Mc Ardle B, Spence JD et al (2012) Imaging atherosclerosis with hybrid $\left[{ }^{18} \mathrm{~F}\right]$ fluorodeoxyglucose positron emission tomography/computed tomography imaging: what Leonardo da Vinci could not see. J Nucl Cardiol. 19(6):1211-1225 